\title{
PRACTICAL PROBLEMS OF ODF EVALUATION
}

\author{
S. PANCHANADEESWARAN AND J. R. HIRSCH \\ Aluminum Company of America, Alcoa Laboratories, USA
}

\section{INTRODUCTION}

Texture measurement has been continuously gaining attention for material characterization. It is well recognized that the 3-dimensional Orientation Distribution Function (ODF) properly describes the texture. Although major improvements have been made in the evaluation of the "true ODF" in recent years, 1 uncertainties still abound in the practical utilization of the available techniques of ODF evaluation from limited pole figure data. The Orientation Distribution Function is most conveniently obtained from reflection pole figures determined by $\mathrm{X}$-ray diffraction. Recently, other techniques have been developed to obtain local pole figures of the microstructure, using transmission electron microscopy (TEM). In either case only partial pole figures are available to extract the ODF.

There are two principal techniques to obtain the ODF, namely: (i) the method of series expansion (SE) in spherical harmonics ${ }^{2}$ and (ii) direct inversion of pole figures (DP). In the SE technique, the ODF is extracted from incomplete pole figures by a commonly used least squares approximation, ${ }^{3}$ or by a recently introduced "positivity" method. ${ }^{4}$ In either case, the accuracy of the resulting ODF is influenced by experimental errors in pole figure measurement as well as by the range of "missing information." The SE method has the advantage, that the ODF is represented in a functional form suitable for evaluation of physical properties. It is, however, necessary to further correct for "ghost" errors. 1 This can be done by Gauss model calculations 6 or also by a positivity method, which uses the same principles as in the case of ODF evaluation from incomplete pole figures. ${ }^{5}$ The Direct Inversion (DR) of pole figures accomplishes the extraction of ODF and the "ghost correction" simultaneously. It should be pointed out, however, that these methods also contain some problems. ${ }^{2}$

The purpose of this paper is twofold. In the first part we address the influence of "missing information" on the accuracy of the ODF. In the second part we discuss the issue of ghost correction and compare "true" SE and DR ODFs. The accuracy of ODF evaluation from limited input data and the effect of the different evaluation and correction methods are analyzed and discussed. 


\section{ODF EVALUATION FROM INCOMPLETE POLE FIGURES}

The series expansion coefficients of the SE-ODF can be calculated from two complete pole figures ${ }^{2}$ up to a degree of series expansion $\ell=22.2$ The same can also be obtained from three incomplete pole figures. In the least squares method ("LS"), an objective function of the integral of the square of the difference between experimental pole intensity and its series expansion is minimized. ${ }^{3}$ In the "positivity" (POS) method this is obtained by an iterative procedure. ${ }^{4}$ In order to evaluate the influence of the "missing" range, model pole figures were regenerated from complete ODFs of a typical aluminum rolling texture (Figure 1). Two types of incomplete pole figures were tested: (a) truncated at constant angle $\alpha$, typical of reflection pole figure (Figure 1a) and (b) truncated at inner big circle (Figure 1b) typical of pole figure data that can be obtained in TEM. The texture used contains rolling components typical of FCC metals, namely Cube, Cube/RD, Goss, $\mathrm{Cu}, \mathrm{S}$ and Bs orientations.

The accuracy of the ODF reproduced from the model pole figures was assessed using the difference texture index defined by

$$
\mathrm{I}_{\mathrm{D}}=\left(\left[\mathrm{C}_{\ell}^{\mathrm{mn}} \operatorname{model}-\mathrm{C}_{\ell}^{\mathrm{mn}}(\mathrm{exp})\right] / 2 \ell+1\right)^{2}
$$
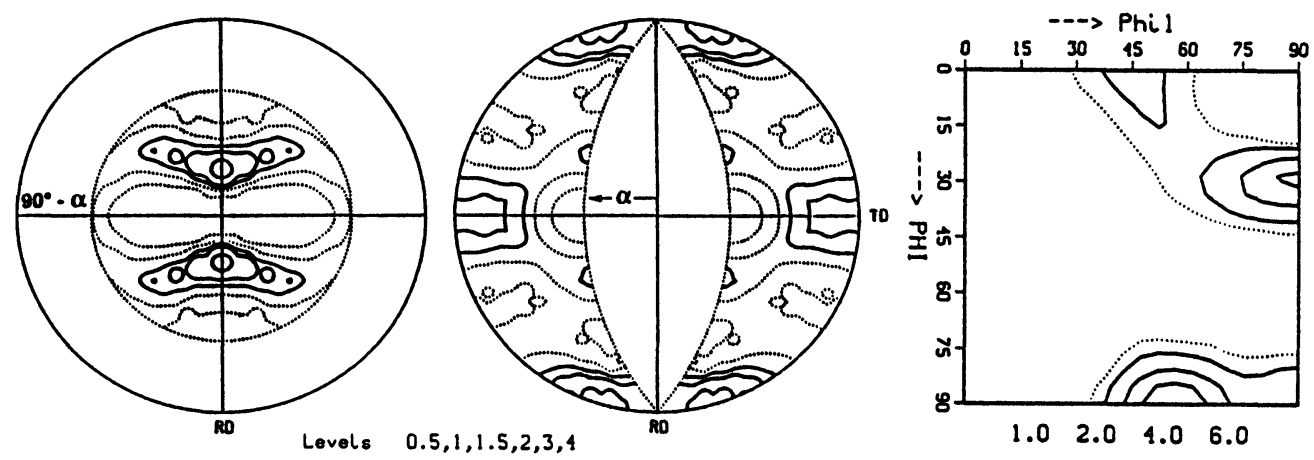

a) Reflection $\alpha_{\max }=65^{\circ}$

b) $\mathrm{TEM} \alpha_{\max }=40^{\circ}$

c) $\operatorname{ODF}\left(\varphi_{2}=45^{\circ}\right.$ section $)$

Figure 1: Input ODF and $\{111\}$ incomplete pole figures used for ODF evaluation

Figures 2 and 3 show the results of ODF evaluations from reflection pole figures by LS and POS methods, respectively, for different values of the truncation angle $\alpha$ (Figure 1a). It may be noticed that in the case of the LS method, there is a sudden loss of accuracy at $\alpha=65^{\circ}$ accompanied by a drastic change in the orientation distribution (Figure $2 \mathrm{c}$ ). This results from the undeterminable $\mathrm{C}_{\ell \mu}^{\mu \mu}$ $(\ell=12,14$ and $18, v=1,2)$ coefficients mainly defined in the missing ranges. The PS method shows a gradual decrease in intensity with decreasing amount of information, maintaining the general shape of the contours (Figure 3). Similar results were obtained for TEM pole figures. 


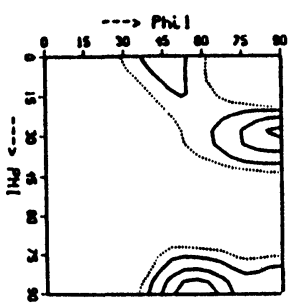

a) $\alpha_{\max }=80^{\circ}$

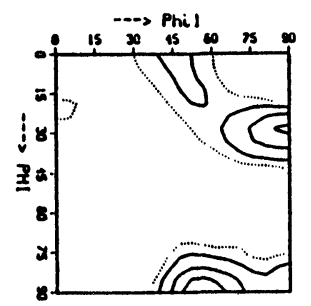

b) $\alpha_{\text {max }}=70^{\circ}$

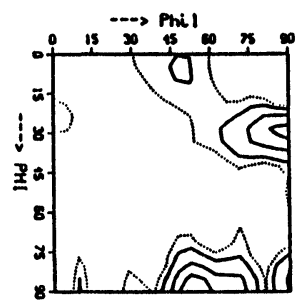

c) $\alpha_{\max }=65^{\circ}$

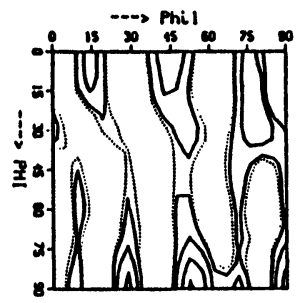

d) $\alpha_{\text {max }}=60^{\circ}$

Figure 2: ODFs recalculated from incomplete reflection pole figure data Least squares (LS) method ( $\varphi_{2}=45^{\circ}$ sections)

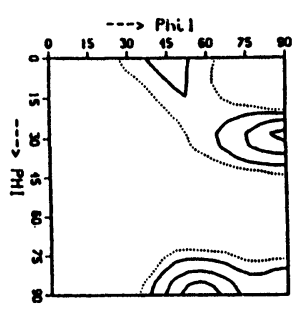

a) $\alpha_{\max }=80^{\circ}$

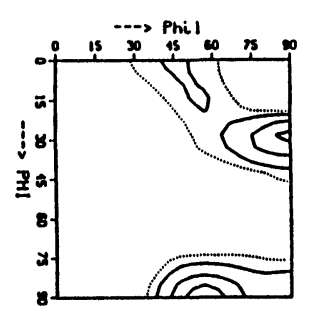

b) $\alpha_{\max }=70^{\circ}$

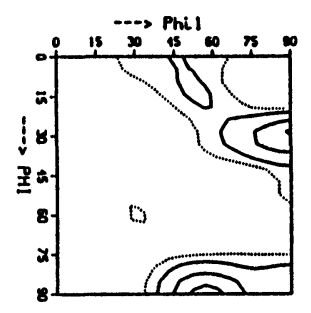

c) $\alpha_{\max }=60^{\circ}$

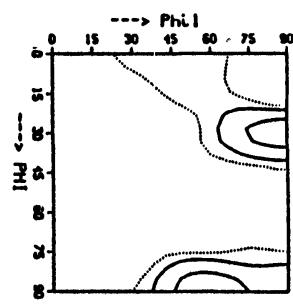

d) $\alpha_{\max }=50^{\circ}$

Figure 3: ODFs recalculated from incomplete reflection pole figure data Positivity (POS) method $\left(\varphi_{2}=0^{\circ}\right.$ sections)

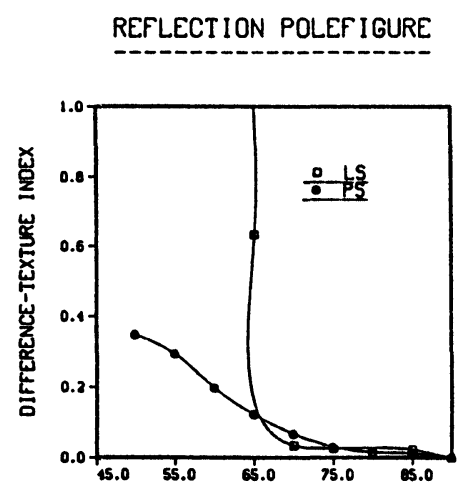

a) MAXIMUM ANGLE RLPHA

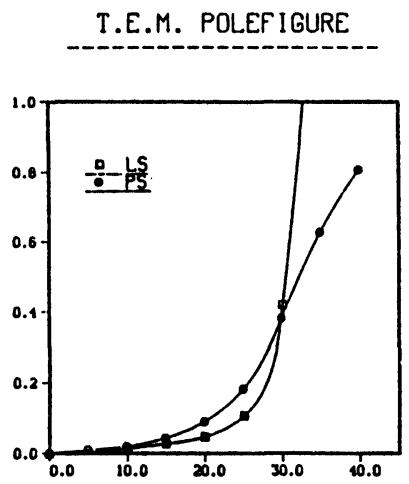

b) maximum angle alpHa
T.E.M. - POLEFIGURE

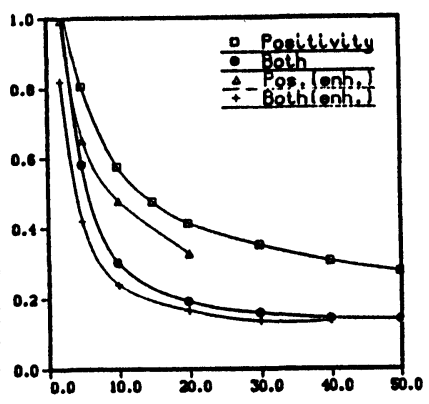

c) MUMBER OF ITERATIONS

Figure 4: Difference texture index $I_{D}$ as function of $\alpha_{\max }(a, b)$ and of iterations (c)
a) from reflection pole figures
b \& c) from TEM pole figures

(c = positivity method, $\alpha_{\max }=40^{\circ}$, "Both" = LS-ODF as initial guess,

"enh" = enhanced iteration method) 
Figure 4 shows the influence of $\alpha$ (i.e., "missing information") and iteration on the difference texture index. As seen above, the loss of accuracy is gradual in the case of the POS method, but is quite drastic in the case of LS method. The results for TEM pole figures (Figure $4 \mathrm{~b}$ ) are similar with a difference that here the "POS" method also shows a sharper rise in the texture index beyond a certain angle.

In the case of the positivity method, the initial guess for the unknown region and the number of iterations strongly influence the final ODF. In Figure 14c, the evolution of the difference texture index is plotted as a function of number of iterations, for TEM pole figures with $\alpha_{\max }=40^{\circ}$. Using the results from the LS method as a starting value for the positivity method (referred to as "both" methods in Figure 4c) instead of a random ODF significantly improves the results. Convergence can further be enhanced by evaluating the differences in series expansion coefficients between iteration steps and multiplying it by a certain factor $\mathrm{f}>1$ (= "enhanced method" in Figure 4c). Figure 5 shows some examples of the ODF evaluations using incomplete TEM pole figure data $\left(\alpha_{\max }=40^{\circ}\right)$ and different ways to improve the results of the positivity method by enhanced iterations and initial guess provided by the LS result.

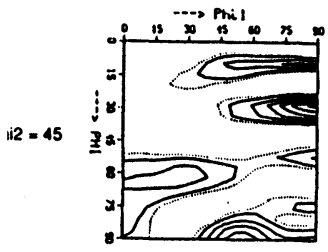

a) Least square method (LS)

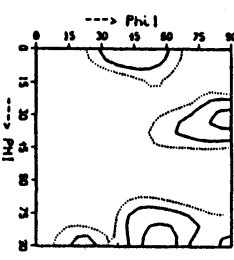

b) Positivity method (5 Iterations)

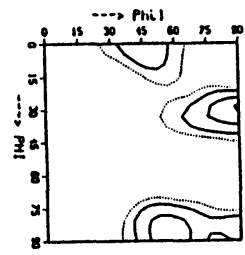

c) Positivity method (30 Iterations)

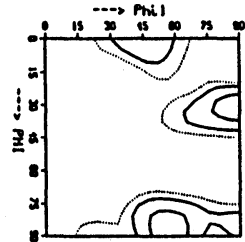

d) Positivity method (10 enhanced Iterations)

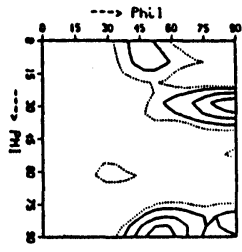

e) Positivity method (5 Iterations) with Initial guess provided by LS method

Figure 5: ODFs $\left(\varphi_{2}=45^{\circ}\right.$ section $)$ calculated from TEM data $\left(\alpha_{\max }=40^{\circ}\right)$

\section{GHOST CORRECTION}

The problem of pole figure inversion cannot be completely solved and the so called "ghost error" often reduces the accuracy of ODF data significantly. In the SE method only even series expansion coefficients can be calculated directly from pole figures. The missing odd coefficients limit the use of ODF since they can seriously falsify the resulting orientation density data by reducing the peak intensities by $10-30 \%$ and creating artificial peaks. ${ }^{1}$ These so called "ghost effects" often make the low intensity areas useless for a detailed analysis. Two SE methods of ghost corrections (i.e., calculation of the missing odd series expansion coefficients) have been established: (i) The positivity (POS) method proposed by Bunge 5 which eliminates the forbidden negative ODF intensities by superimposing an appropriate odd SE ODF and (ii) The Gauss model (GM) method proposed by Lücke et al. 6 which reproduces the even ODF by a model ODF composed of a few model components with Gaussian scatterings. Both methods correct the experimental (even) ODF by adding newly generated odd coefficients to the existing even ones. 
The results of such ghost corrected ODFs are shown in Figure $6 \mathrm{~b}$ and $\mathrm{c}$ for a sharp $\mathrm{Al}$ rolling texture where strong ghost effects are observed (Figure 6a). Both methods achieve a significant improvement of the data and efficiently reduce the ghost error. In spite of the different approaches (the POS method makes use of low intensity regions while the GM uses the texture peaks), the results are surprisingly similar which suggest that both methods approximate the true ODF to a rather similar degree.

The problem of ghost error is less severe in direct methods ("DR") of ODF evaluation from pole figures. ${ }^{1}$ The solution can be expected to be closer to the true ODF especially when large zero ranges are present since these methods make implicit use of the positivity condition. This is usually the case for sharp textures (where the ghost problem is worst in SE ODFs). Another advantage is that the peak intensities are not reduced due to truncation as in the SE methods.

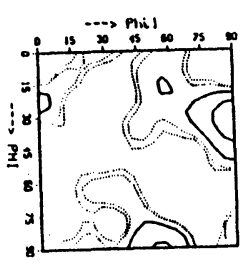

a) Incomplete SE-ODF $\left(C_{\text {odd }}=0\right)$

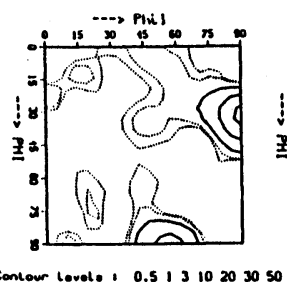

b) Complete SE-ODF (zero range method)

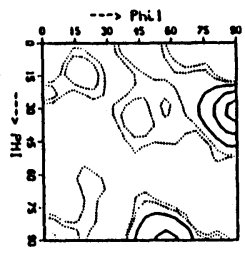

C) Complete SE-ODF (Gauss model analysis)

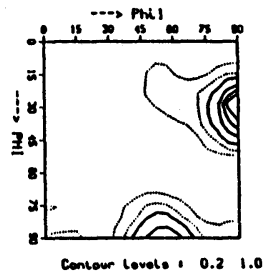

d) DR-ODF (WIMV method)

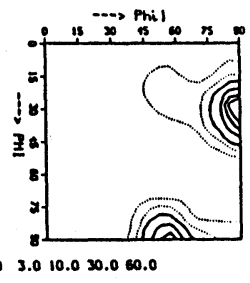

e) DR-ODF (Pawlik) Posplech method)

Figure 6: $\mathrm{ODF}$ ( $\varphi_{2}=45^{\circ}$ section) of a strong $\mathrm{Al}$ rolling texture, evaluated by series expansion (SE) (b,c = ghost corrected) and direct (DR) methods (d,e)

a) Incomplete SE-ODF $\left(C_{\text {odd }}=0\right)$, b) Complete SE-ODF (zero range method [5]), c) Complete SE-ODF (Gauss model analysis [6]), d) DR-ODF (WIMV method [8]), e) DR-ODF (ADC method [9]) (dotted lines correspond to intensity levels $\leq 1$ )

In Figures $6 \mathrm{~d}$ and $6 \mathrm{e}$, the results of two direct methods are shown, using the WIMV algorithm ${ }^{8}$ and the ADC method. ${ }^{9}$ Ten iteration steps were used and the results are quite similar. Both reveal the sharp rolling texture with much stronger orientation densities than the SE ODF (Figures 6a-c). It is a characteristic effect for the DR algorithm to prefer the strong peaks but it neglects small intensities. 10 For this reason the SE methods appear to be more suitable for analyzing low intensity regions of the ODF. In the example presented in Figure $6 \mathrm{~b}, \mathrm{c}$, the Cube-RD scattering (which reveals the texture evolution) is best visible in the (ghost corrected) SE-ODFs. So, for practical cases, the DR method seems to be better when the main (i.e., strong) features of the texture are to be analyzed whereas the $\mathrm{SE}$ method is more suitable for the investigation of texture details in low intensity areas (after proper ghost correction). 


\section{SUMMARY}

Current methods of ODF evaluation from incomplete pole figures and methods of ghost correction were tested for their practical use. The accuracy of ODF data evaluation is analyzed as functions of the amount of (reduced) pole figure information due to the necessity to work with limited experimental data available in $\mathrm{X}$-ray reflection mode or TEM local pole figure analysis. The different response of the two methods using least square and positivity conditions is analyzed quantitatively. It is shown that a reasonably good ODF evaluation can be obtained from three incomplete pole figures obtained in reflection mode up to $\alpha_{\max }=65^{\circ}$ or up to $40^{\circ}$ great circle area in TEM transmission mode. The results can be improved when both methods are combined and iteration is enhanced.

Ghost correction methods for the series expansion methods using Gauss model functions and positivity conditions are tested. It is shown that these two different approaches lead to very similar results. Also the two direct methods tested (WIMV and ADC) reveal similar (ghost free) ODFs. In comparison to series expansion ODFs the direct methods emphasize the main features and high intensities of the texture while the series expansion methods better reflect texture effects in low intensity regions.

\section{ACKNOWLEDGMENT}

The authors thank J. S. Kallend and U. F. Kocks for making available the WIMV program and K. Pawlik and J. Pospiech for their ADC program of ODF evaluation. The cooperation with the Alcoa X-ray laboratory personnel who provided experimental data is gratefully acknowledged. Thanks to C. L. Schall for carefully preparing the manuscript.

\section{REFERENCES}

1. H. R. Wenk, H. J. Bunge, J. S. Kallend, K. Lücke, S. Matthies, J. Pospiech, P. v. Houtte, Proceedings ICOTOM8, Santa Fe, New Mexico,, ed. J. S. Kallend, G. Gottstein, TMS, 1988, p. 17.

2. H. J. Bunge, "Texture Analysis in Materials science," Butterworths, London, 1982.

3. J. Jura, J. Pospiech, H. J. Bunge, Metallurgica, 24, 1976, p. 111.

4. M. Dahms, H. J. Bunge, Proceedings ICOTOM8, Santa Fe, New Mexico, ed. J. S. Kallend, G. Gottstein, TMS, 1988, p. 79.

5. H, J. Bunge, Proceedings ICOTOM8, Santa Fe, New Mexico, ed. J. S. Kallend, G. Gottstein, TMS, 1988, p. 69.

6. K. Lücke, J. Pospiech, K. H. Virnich, J. Jura, Acta Met , 19, 1981, p. 167.

7. K. H. Virnich, J. Pospiech, A. Flemmer, K. Lücke, Proceedings ICOTOM5, Aachen, 1978, p. 129.

8. S. Matthies, Proceedings ICOTOM8, Santa Fe, New Mexico, ed. J. S. Kallend, G. Gottstein, TMS, 1988, pg. 37.

9. K. Pawlik, J. Pospiech, K. Lücke, Proceedings ICOTOM8, Santa Fe, New Mexico, ed. J. S. Kallend, G. Gottstein, TMS, 1988, pg. 105.

10. J. Pospiech, Proceedings, ICOTOM8, Santa Fe, New Mexico, ed. J. S. Kallend, G. Gottstein, TMS, 1988, pg. 93. 